\title{
LOEB EXTENSION AND LOEB EQUIVALENCE
}

\author{
ROBERT M. ANDERSON, HAOSUI DUANMU, DAVID SCHRITTESSER, \\ AND WILLIAM WEISS \\ (Communicated by Heike Mildenberger)
}

\begin{abstract}
In [J. London Math. Soc. 69 (2004), pp. 258-272] Keisler and Sun leave open several questions regarding Loeb equivalence between internal probability spaces; specifically, whether under certain conditions, the Loeb measure construction applied to two such spaces gives rise to the same measure. We present answers to two of these questions, by giving two examples of probability spaces. Moreover, we reduce their third question to the following: Is the internal algebra generated by the union of two Loeb equivalent internal algebras a subset of their common Loeb extension? We also present a sufficient condition for a positive answer to this question.
\end{abstract}

\section{INTRODUCTION}

The Loeb measure construction ([Loe75]) has many fruitful applications in various areas in mathematics such as probability theory (see And76, AR78, Per81, [Kei84, Sto86], Lin90, Lin04, DRW18, ADS18] etc), statistical decision theory (see [DR18]), potential theory ([Loe76]), mathematical physics (see [AHKFL86]) and mathematical economics (see [BR74, Kha74, BR75], KR75], Kha76, Ras78, [Emm84, And85, And88, And91, Sun96], AKRS97], Sun99], KS99], AKS03], [Rau07, DS07, AR08, Sun16, DQS18, CHLS19, among others) 11 These applications are made possible by a well-developed theory of integration (see Loe75. and And76]), representation of measures (see And82 and Sti97]) and a Fubini theorem for Loeb measures (see Kei84]). KS04 made an important contribution to these elements of the theory of Loeb measure, and also left four open questions. In this paper we address the first three of these.

Given an internal probability space $(\Omega, \mathcal{F}, \mu)$, its Loeb extension is defined to be the countably additive probability space $(\Omega, \overline{\mathcal{F}}, \bar{\mu})$, where $\overline{\mathcal{F}}$ consists of all sets $B \subset \Omega$ such that

$$
\sup \{\operatorname{st}(\mu(A)): B \supset A \in \mathcal{F}\}=\inf \{\operatorname{st}(\mu(C)): B \subset C \in \mathcal{F}\}
$$

and $\bar{\mu}(B)$ is defined to be the above supremum. KS04 introduced the following definition to compare two internal probability spaces.

Received by the editors July 29, 2020, and, in revised form, December 7, 2020.

2020 Mathematics Subject Classification. Primary 28E05; Secondary 03H05, 26E35.

${ }^{1}$ BR74], Kha74], BR75], KR75] and Kha76] predated the Loeb measure construction. They relied on a careful analysis of the close relationship between the discrete and measure-theoretic properties of hyperfinite sets that the Loeb measure construction so perfectly captures. The arguments in these papers led, via a Loeb space argument, to a very general and completely elementary argument given in And78.

(C)2021 by the authors under Creative Commons Attribution-Noncommercial 3.0 License (CC BY NC 3.0) 
Definition 1.1. Let $\mathcal{M}=(\Omega, \mathcal{F}, \mu)$ and $\mathcal{N}=(\Omega, \mathcal{G}, \nu)$ be two internal probability spaces. We say $\mathcal{N}$ Loeb extends $\mathcal{M}$ if $\overline{\mathcal{G}} \supset \overline{\mathcal{F}}$ and $\bar{\nu}$ extends $\bar{\mu}$ as a function. We say $\mathcal{N}$ is Loeb equivalent to $\mathcal{M}$ if $\overline{\mathcal{F}}=\overline{\mathcal{G}}$ and $\bar{\nu}=\bar{\mu}$.

If $\mathcal{F} \subset \mathcal{G}$ and $\nu$ extends $\mu$ as a function, then it is clear that $\mathcal{N}$ Loeb extends $\mathcal{M}$. However, as pointed out by [KS04, without the assumption $\mathcal{F} \subset \mathcal{G}$, it is difficult to make any assertions about the relationship between their Loeb extensions. These difficulties are addresses by the following three questions posed in KS04.

To state these question, let $\mathcal{N}$ and $\mathcal{M}$ be internal probability spaces. Moreover let us fix the following terminology: We say an internal probability space $(\Omega, \mathcal{F}, \mu)$ is hyperfinite if $\mathcal{F}$ is hyperfinite. We emphasize that as in KS04, we do not require $\Omega$ to be hyperfinite.

Question 1. Suppose $\mathcal{N}$ Loeb extends $\mathcal{M}$. Must $\mathcal{N}$ have an internal subspace that is Loeb equivalent to $\mathcal{M}$ ? What if $\mathcal{M}$ is assumed to be hyperfinite?

Question 2. Suppose $\mathcal{N}$ Loeb extends $\mathcal{M}$ and $\mathcal{N}$ is hyperfinite. Must $\mathcal{M}$ be Loeb equivalent to a hyperfinite probability space?

Question 3. Suppose $\mathcal{M}=(\Omega, \mathcal{F}, \mu)$ is Loeb equivalent to $\mathcal{N}=(\Omega, \mathcal{G}, \nu)$ and let $\mathcal{H}$ be the internal algebra generated by $\mathcal{F} \cup \mathcal{G}$. Must there be an internal probability measure $P$ on $\mathcal{H}$ such that $\mathcal{M}$ is Loeb equivalent to $(\Omega, \mathcal{H}, P)$ ? What if $\mathcal{M}$ and $\mathcal{N}$ are assumed to be hyperfinite?

In this paper give complete solutions to the first two of these questions, as well as a partial solution to the third.

\section{Counter-examples For the First two Questions}

We start this section by introducing the following theorem which provides a useful characterization of Loeb extension. The first part of the theorem is cited from [KS04, Lemma. 4.3].

Theorem 2.1. Let $\mathcal{M}=(\Omega, \mathcal{F}, \mu)$ and $\mathcal{N}=(\Omega, \mathcal{G}, \nu)$ be two internal probability spaces.

- $\mathcal{N}$ Loeb extends $\mathcal{M}$ if and only if for every $B \in \mathcal{F}$ there exists $C \in \mathcal{G}$ such that $B \subset C$ and $\nu(C) \approx \mu(B)$.

- $\mathcal{N}$ Loeb extends $\mathcal{M}$ if and only if for every $B \in \mathcal{F}$ there exists $C \in \mathcal{G}$ such that $C \subset B$ and $\nu(C) \approx \mu(B)$.

Proof. The first statement is cited directly from [KS04, Lemma. 4.3]. We give a proof of the second statement for completeness. Suppose $\mathcal{N}$ Loeb extends $\mathcal{M}$ and pick $B \in \mathcal{F}$. Then there exists $C \in \mathcal{G}$ such that $\Omega \backslash B \subset C$ and $\nu(C) \approx \mu(\Omega \backslash B)$. Thus, we have $\Omega \backslash C \subset B$ and $\nu(\Omega \backslash C) \approx \mu(B)$. Conversely, for every $B \in \mathcal{F}$, there exists $C \in \mathcal{G}$ such that $C \subset \Omega \backslash B$ and $\nu(C) \approx \mu(\Omega \backslash B)$. Hence, we have $B \subset \Omega \backslash C$ and $\nu(\Omega \backslash C) \approx \mu(B)$. By the first statement, we know that $\mathcal{N}$ Loeb extends $\mathcal{M}$.

The following theorem gives a condition which is necessary in order that $\mathcal{N}$ contain an internal subspace which is Loeb equivalent to $\mathcal{M}$.

Theorem 2.2. Suppose $\mathcal{N}$ Loeb extends $\mathcal{M}$ and $\mathcal{N}=(\Omega, \mathcal{G}, \nu)$ has an internal subspace that is Loeb equivalent to $\mathcal{M}=(\Omega, \mathcal{F}, \mu)$. Then, for every $A \in \mathcal{F}$ there exists $A^{\prime} \in \mathcal{F}$ such that $A^{\prime} \subset B \subset A$ for some $B \in \mathcal{G}$ and $\mu\left(A^{\prime}\right) \approx \mu(A)$. If $\mathcal{N}$ is hyperfinite, then $B$ can be taken to be $\bigcup\{C \in \mathcal{G}: C \subset A\}$. 
Proof. Let $\mathcal{N}^{\prime}=\left(\Omega, \mathcal{G}^{\prime}, \nu^{\prime}\right)$, where $\nu^{\prime}$ is the restriction of $\nu$ to $\mathcal{G}^{\prime}$, be an internal subspace of $\mathcal{N}$ that is Loeb equivalent to $\mathcal{M}$. Pick $A \in \mathcal{F}$. By Theorem 2.1, there exists $B \in \mathcal{G}$ such that $B \subset A$ and $\nu(B) \approx \mu(A)$. By Theorem 2.1 again, we know that there exists $A^{\prime} \in \mathcal{F}$ such that $A^{\prime} \subset B$ and $\mu\left(A^{\prime}\right) \approx \nu(B)$.

Question 1 asks whether the converse of Theorem 2.2 is true. We provide a counter-example below.

Example 2.3. Let $N=\frac{1}{K !}$ for some $K \in{ }^{*} \mathbb{N} \backslash \mathbb{N}$ and let $\Omega=\left\{\frac{1}{N}, \frac{2}{N}, \ldots, 1\right\}$. Then $\Omega$ includes the set of all rational numbers in $[0,1]$ as a subset. Let $P$ denote the uniform hyperfinite probability measure on $\Omega$, that is, $P(\{\omega\})=\frac{1}{N}$ for every $\omega \in \Omega$. Let

$$
\mathcal{F}=\left\{\emptyset, \Omega,\left\{\frac{1}{N}, \frac{2}{N}, \ldots, \frac{1}{2}\right\},\left\{\frac{1}{2}+\frac{1}{N}, \ldots, 1\right\}\right\}
$$

and let $\mathcal{G}$ be the internal algebra generated by

$$
\left\{\left\{\frac{1}{N}, \frac{2}{N}, \ldots, \frac{1}{2}-\frac{2}{N}\right\},\left\{\frac{1}{2}-\frac{1}{N}, \frac{1}{2}, \frac{1}{2}+\frac{1}{N}\right\},\left\{\frac{1}{2}+\frac{2}{N}, \ldots, 1\right\}\right\} .
$$

Let $\mu$ and $\nu$ be restrictions of $P$ on $\mathcal{F}$ and $\mathcal{G}$, respectively. Finally, let $\mathcal{M}=(\Omega, \mathcal{F}, \mu)$ and $\mathcal{N}=(\Omega, \mathcal{G}, \nu)$. By Theorem 2.1. it is clear that $\mathcal{N}$ Loeb extends $\mathcal{M}$. On the other hand, the Loeb $\sigma$-algebra $\overline{\mathcal{F}}$ generated from $\mathcal{F}$ is the same as $\mathcal{F}$. Thus, $\overline{\mathcal{F}}$ does not contain any element in $\left\{\left\{\frac{1}{N}, \frac{2}{N}, \ldots, \frac{1}{2}-\frac{2}{N}\right\},\left\{\frac{1}{2}-\frac{1}{N}, \frac{1}{2}, \frac{1}{2}+\frac{1}{N}\right\},\left\{\frac{1}{2}+\frac{2}{N}, \ldots, 1\right\}\right\}$, hence $\mathcal{M}$ is not Loeb equivalent to any internal subset of $\mathcal{N}$.

Since the answer to Question 1 is negative, we now turn our attention to Question 2. The following example shows that the answer to Question 2 is also negative.

Example 2.4. Let $N$ be an element of ${ }^{*} \mathbb{N} \backslash \mathbb{N}$ and let $\Omega={ }^{*}[0,1]$. Let $\lambda$ denote the Lebesgue measure on $[0,1]$. Let $\mathcal{F}$ be the internal algebra generated by ${ }^{*}\left[0, \frac{1}{2}\right),{ }^{*}\left[\frac{1}{2}, 1\right]$ and all $\{a\}$ for $a \in \in^{*}\left[\frac{1}{2}-\frac{1}{N}, \frac{1}{2}+\frac{1}{N}\right]$. Clearly, $\mathcal{F}$ is not hyperfinite. Moreover, a subset of * $\left[\frac{1}{2}-\frac{1}{N}, \frac{1}{2}+\frac{1}{N}\right]$ is an element of $\mathcal{F}$ if and only if it is hyperfinite. Let $\mathcal{G}$ be the internal algebra generated by $\left\{{ }^{*}\left[0, \frac{1}{2}-\frac{1}{N}\right),{ }^{*}\left[\frac{1}{2}-\frac{1}{N}, \frac{1}{2}+\frac{1}{N}\right],{ }^{*}\left(\frac{1}{2}+\frac{1}{N}, 1\right]\right\}$. Let $\mu$ and $\nu$ be the restrictions of ${ }^{*} \lambda$ on $\mathcal{F}$ and $\mathcal{G}$, respectively. Finally, let $\mathcal{M}=(\Omega, \mathcal{F}, \mu)$ and $\mathcal{N}=(\Omega, \mathcal{G}, \nu)$. It is clear that $\mathcal{N}$ is hyperfinite and, by Theorem 2.1, $\mathcal{N}$ Loeb extends $\mathcal{M}$. Moreover, we have the following lemma:

Lemma 2.5. For every internal set $F \in \overline{\mathcal{F}}, \bar{\mu}(F)=0$ if and only if $F$ is hyperfinite.

Proof. Pick $F \in \overline{\mathcal{F}}$. Clearly, if $F$ is hyperfinite, then $\bar{\mu}(F)=0$. If $\bar{\mu}(F)=0$, then there must exist $F^{\prime} \in \mathcal{F}$ such that $F \subset F^{\prime}$ and $\mu\left(F^{\prime}\right) \approx 0$. By the construction of $\mathcal{F}, F^{\prime}$ must be hyperfinite. As $F$ is internal, $F$ must be hyperfinite.

We now show that $\mathcal{M}$ is not Loeb equivalent to any hyperfinite probability space. Suppose not. Let $\mathcal{N}^{\prime}=\left(\Omega, \mathcal{G}^{\prime}, P\right)$ be a hyperfinite probability space that is Loeb equivalent to $\mathcal{M}$. For every $a \in \in^{*}\left[\frac{1}{2}-\frac{1}{N}, \frac{1}{2}+\frac{1}{N}\right]$, by Theorem [2.1, there exists $A_{a} \in \mathcal{G}^{\prime}$ such that $a \in A_{a}$ and $P\left(A_{a}\right) \approx 0$. Pick $n \in \mathbb{N}$ and let

$\mathcal{A}_{n}=\left\{A \in \mathcal{G}^{\prime}:\left(P(A)<\frac{1}{n}\right) \wedge\left(a \in A\right.\right.$ for *infinitely many $\left.\left.a \in \in^{*}\left[\frac{1}{2}-\frac{1}{N}, \frac{1}{2}+\frac{1}{N}\right]\right)\right\}$

Note that there are *infinitely many $a \in *^{*}\left[\frac{1}{2}-\frac{1}{N}, \frac{1}{2}+\frac{1}{N}\right]$ and hyperfinitely many $A \in \mathcal{G}^{\prime}$ with $P(A)<\frac{1}{n}$. As each $a \in *^{*}\left[\frac{1}{2}-\frac{1}{N}, \frac{1}{2}+\frac{1}{N}\right]$ must be contained in some $A \in \mathcal{G}^{\prime}$ such that $P(A)<\frac{1}{n}$, by the transfer of the pigeonhole principle, $\mathcal{A}_{n}$ is non-empty for every $n \in \mathbb{N}$. By the saturation principle, there exists an internal 
$A_{0}$ such that $P\left(A_{0}\right) \approx 0$ and $A_{0}$ contains $*$ infinitely many $a \in *^{*}\left[\frac{1}{2}-\frac{1}{N}, \frac{1}{2}+\frac{1}{N}\right]$. As $\mathcal{N}^{\prime}$ is Loeb equivalent to $\mathcal{M}$, we know that $\bar{\mu}\left(A_{0}\right)=0$. This, however, contradicts Lemma 2.5, hence we conclude that $\mathcal{M}$ is not Loeb equivalent to any hyperfinite probability space.

In summary, both Question 1 and Question 2 have negative answers. In general, if $\mathcal{N}$ Loeb extends $\mathcal{M}$, it needn't be the case that $\mathcal{M}$ is equivalent to a subspace of $\mathcal{N}$.

\section{A REFORMUlation OF THE THIRD QUESTION}

In this and the following sections, we give a partial answers to Question 3 In this section, we reduce Question 3 to the following question in the hyperfinite case: for hyperfinite spaces, is the internal algebra generated by the union of two Loeb equivalent internal algebras a subset of the Loeb extension of one of the two generating algebras?

Let $(\Omega, \mathcal{F}, \mu)$ be an internal probability space and let $\mathcal{G}$ be an internal algebra on $\Omega$. The following theorem shows that $\mathcal{G} \subset \overline{\mathcal{F}}$ if and only if it is possible to define an internal measure $\nu$ on $(\Omega, \mathcal{G})$ such that $(\Omega, \mathcal{F}, \mu)$ Loeb extends $(\Omega, \mathcal{G}, \nu)$.

Theorem 3.1. Suppose $\mathcal{M}=(\Omega, \mathcal{F}, \mu)$ is a hyperfinite probability space and $\mathcal{G}$ is a hyperfinite algebra on $\Omega$. Then $(\Omega, \mathcal{F}, \mu)$ Loeb extends $(\Omega, \mathcal{G}, P)$ for some internal probability measure $P$ if and only if $\mathcal{G} \subset \overline{\mathcal{F}}$.

Proof. Suppose, for some internal probability measure $P,(\Omega, \mathcal{F}, \mu)$ Loeb extends $(\Omega, \mathcal{G}, P)$. Then we have $\overline{\mathcal{G}} \subset \overline{\mathcal{F}}$ which implies that $\mathcal{G} \subset \overline{\mathcal{F}}$.

Now suppose $\mathcal{G} \subset \overline{\mathcal{F}}$. As $\mathcal{F}$ is hyperfinite, by the transfer principle, there exists an internal subset $\mathcal{F}_{0}$ of $\mathcal{F}$ such that

(1) $\mathcal{F}_{0}$ is a *partition of $\Omega$

(2) Each $A \in \mathcal{F}_{0}$ is an atom of $\mathcal{F}$, i.e., for any non-empty $E \in \mathcal{F}, E \subset A \Rightarrow$ $E=A$.

Any $F \in \mathcal{F}$ is a hyperfinite union of elements from $\mathcal{F}_{0}$, i.e., $\mathcal{F}_{0}$ internally generates the algebra $\mathcal{F}$. Similarly, there exists an internal subset $\mathcal{G}_{0}$ of $\mathcal{G}$ such that

(1) $\mathcal{G}_{0}$ is a *partition of $\Omega$

(2) Each $A \in \mathcal{G}_{0}$ is an atom in $\mathcal{G}$.

Let $\mathcal{U}=\left\{A \cap B: A \in \mathcal{F}_{0}, B \in \mathcal{G}_{0}, A \cap B \neq \emptyset\right\}$. It is easy to see that $\mathcal{U}$ forms a * partition of $\Omega$ and every element in the algebra generated by $\mathcal{F} \cup \mathcal{G}$ can be written as a hyperfinite union of elements in $\mathcal{U}$. For $F \in \mathcal{F}_{0}$, let $\mathcal{U}_{F}=\{F \cap B: B \in$ $\left.\mathcal{G}_{0}, B \cap F \neq \emptyset\right\}$. Then $\mathcal{U}=\bigcup_{F \in \mathcal{F}_{0}} \mathcal{U}_{F}$ and $\mathcal{U}_{F}$ is hyperfinite for every $F \in \mathcal{F}_{0}$. We now define a function $P^{\prime}: \mathcal{U} \rightarrow{ }^{*}[0,1]$. For every $U \in \mathcal{U}, U \in \mathcal{U}_{F_{0}}$ for exactly one $F_{0} \in \mathcal{F}_{0}$, let $P^{\prime}(U)=\frac{\mu\left(F_{0}\right)}{\left|\mathcal{U}_{F_{0}}\right|}$ where $\left|\mathcal{U}_{F_{0}}\right|$ denotes the internal cardinality of $\mathcal{U}_{F_{0}}$.

Claim 3.2. For every $A \in \mathcal{F}, \mu(A)=\sum_{U \in \mathcal{U}, U \subset A} P^{\prime}(U)$.

Proof. Pick $A \in \mathcal{F}$. Let $\mathcal{F}_{A}=\left\{F \in \mathcal{F}_{0}: F \subset A\right\}$. Every $U \in \mathcal{U}$ such that $U \subset A$ is an element of exactly one element in $\mathcal{F}_{A}$. Moreover, as $\mathcal{U}$ forms a *partition of $\Omega$, an element in $\mathcal{U}$ is either a subset of $A$ or disjoint from $A$. Thus, we have

$$
\mu(A)=\sum_{F \in \mathcal{F}_{A}} \mu(F)=\sum_{F \in \mathcal{F}_{A}} \sum_{U \in \mathcal{U}, U \subset F} \frac{\mu(F)}{\left|\mathcal{U}_{F}\right|}=\sum_{U \in \mathcal{U}, U \subset A} P^{\prime}(U) .
$$


Define $P: \mathcal{G} \rightarrow{ }^{*}[0,1]$ by letting $P(G)=\sum_{U \in \mathcal{U}, U \subset G} P^{\prime}(U)$.

Claim 3.3. $P$ is an internal probability measure on $(\Omega, \mathcal{G})$.

Proof. Clearly we have $P(\emptyset)=0$ and $P(\Omega)=1$. Let $G_{1}, G_{2} \in \mathcal{G}$ be two disjoint sets. Let $U_{0} \subset G_{1} \cup G_{2}$ be an element of $\mathcal{U}$. As $\mathcal{U}$ forms a * partition of $\Omega$ and both $G_{1}$ and $G_{2}$ can be written as a hyperfinite union of elements in $\mathcal{U}$, we can conclude that $U_{0}$ is either a subset of $G_{1}$ or a subset of $G_{2}$. Thus, we have

$$
\begin{aligned}
P\left(G_{1} \cup G_{2}\right) & =\sum_{U \in \mathcal{U}, U \subset G_{1} \cup G_{2}} P^{\prime}(U) \\
& =\sum_{V \in \mathcal{U}, V \subset G_{1}} P^{\prime}(V)+\sum_{E \in \mathcal{U}, E \subset G_{2}} P^{\prime}(E) \\
& =P\left(G_{1}\right)+P\left(G_{2}\right) .
\end{aligned}
$$

We now show that $(\Omega, \mathcal{F}, \mu)$ Loeb extends $(\Omega, \mathcal{G}, P)$. Pick $G \in \mathcal{G}$. Let $G_{i}=$ $\bigcup\{F \in \mathcal{F}: F \subset G\}$ and let $G_{o}=\bigcap\{F \in \mathcal{F}: G \subset F\}$. As $\mathcal{F}$ is hyperfinite, both $G_{i}$ and $G_{o}$ are elements of $\mathcal{F}$. Moreover, as $\mathcal{G} \subset \overline{\mathcal{F}}$, we have $\mu\left(G_{i}\right) \approx \mu\left(G_{o}\right)$, which implies that $\bar{\mu}(G)=\operatorname{st}\left(\mu\left(G_{i}\right)\right)$. As $G_{i} \subset G \subset G_{o}$, we have $\sum_{U \in \mathcal{U}, U \subset G_{i}} P^{\prime}(U) \leq$ $P(G) \leq \sum_{U \in \mathcal{U}, U \subset G_{o}} P^{\prime}(U)$. By Claim 3.2, we have $\sum_{U \in \mathcal{U}, U \subset G_{i}} P^{\prime}(U)=\mu\left(G_{i}\right)$ and $\sum_{U \in \mathcal{U}, U \subset G_{o}} P^{\prime}(U)=\mu\left(G_{o}\right)$. Thus, we can conclude that $\bar{P}(G)=\operatorname{st}(P(G))=$ st $\left(\mu\left(G_{i}\right)\right)=\bar{\mu}(G)$, completing the proof.

The following theorem gives a partial answer to Question 3 .

Theorem 3.4. Let $(\Omega, \mathcal{F}, \mu)$ be a hyperfinite probability space and let $\mathcal{G}$ be a hyperfinite algebra on $\Omega$. Let $\mathcal{H}$ be the internal algebra generated by $\mathcal{F} \cup \mathcal{G}$. Then $(\Omega, \mathcal{H}, P)$ is Loeb equivalent to $(\Omega, \mathcal{F}, \mu)$ for some internal probability measure $P$ if and only if $\mathcal{H} \subset \overline{\mathcal{F}}$.

Proof. Suppose there exists an internal probability measure $P$ such that $(\Omega, \mathcal{H}, P)$ is Loeb equivalent to $(\Omega, \mathcal{F}, \mu)$. Then we have $\overline{\mathcal{H}}=\overline{\mathcal{F}}$ which implies that $\mathcal{H} \subset \overline{\mathcal{F}}$.

Now suppose $\mathcal{H} \subset \overline{\mathcal{F}}$. By Theorem 3.1, there exists an internal probability measure $P$ on $(\Omega, \mathcal{H})$ such that $(\Omega, \mathcal{F}, \mu)$ Loeb extends $(\Omega, \mathcal{H}, P)$. Thus, we have $P(F) \approx \mu(F)$ for every $F \in \mathcal{F}$. By Theorem 2.1 . $(\Omega, \mathcal{H}, P)$ Loeb extends $(\Omega, \mathcal{F}, \mu)$ and we have the desired result.

It is natural to ask if Theorem 3.4 remains valid without the hyperfinite assumption.

Open Problem 1. Let $\mathcal{M}=(\Omega, \mathcal{F}, \mu)$ and $\mathcal{N}=(\Omega, \mathcal{G}, \nu)$ be two internal probability spaces that are not hyperfinite. Let $\mathcal{H}$ be the internal algebra generated by $\mathcal{F} \cup \mathcal{G}$. Suppose $\mathcal{M}$ is Loeb equivalent to $\mathcal{N}$, and $\mathcal{H} \subset \overline{\mathcal{F}}$. Must there be an internal probability measure $P$ on $\mathcal{H}$ such that $\mathcal{M}$ is Loeb equivalent to $(\Omega, \mathcal{H}, P)$ ?

\section{Addtional Results on the third question}

By Theorem 3.4, a positive answer to the following question leads to a positive answer to Question [3] in the hyperfinite case.

Open Problem 2. Let $(\Omega, \mathcal{F}, \mu)$ be a hyperfinite probability space and let $\mathcal{G}$ be a hyperfinite algebra on $\Omega$ such that $\mathcal{G} \subset \overline{\mathcal{F}}$. Let $\mathcal{H}$ be the hyperfinite algebra generated by $\mathcal{F} \cup \mathcal{G}$. Is $\mathcal{H} \subset \overline{\mathcal{F}}$ ? 
We suspect the answer to the question posed in Open Problem 2 is negative. However, we shall momentarily give a sufficient condition for a positive answer.

Throughout this section, let $\mathcal{M}=(\Omega, \mathcal{F}, \mu)$ and $\mathcal{N}=(\Omega, \mathcal{G}, \nu)$ be two hyperfinite probability spaces and let $\mathcal{H}$ be the internal algebra generated by $\mathcal{F} \cup \mathcal{G}$. We use the same notation as in the proof of Theorem 3.1. Throughout this section, let $\mathcal{F}_{0}$ be a set of atoms for $\mathcal{F}$, i.e., an internal subset of $\mathcal{F}$ such that

(1) $\mathcal{F}_{0}$ is a *partition of $\Omega$

(2) For every $A \in \mathcal{F}_{0}$, if there exists non-empty $E \in \mathcal{F}$ such that $E \subset A$, then $E=A$.

Similarly, let $\mathcal{G}_{0}$ be a set of atoms for $\mathcal{G}$, i.e., an internal subset of $\mathcal{G}$ such that

(1) $\mathcal{G}_{0}$ is a ${ }^{*}$ partition of $\Omega$

(2) For every $A \in \mathcal{G}_{0}$, if there exists non-empty $E \in \mathcal{G}$ such that $E \subset A$, then $E=A$.

Let $\mathcal{U}=\left\{A \cap B: A \in \mathcal{F}_{0}, B \in \mathcal{G}_{0}, A \cap B \neq \emptyset\right\}$. Then $\mathcal{U}$ forms a *partition of $\Omega$ and every element in $\mathcal{H}$ can be written as a hyperfinite union of elements in $\mathcal{U}$. Assuming $\mathcal{G} \subset \overline{\mathcal{F}}$, it follows that $\mathcal{U} \subset \overline{\mathcal{F}}$. However, it is not clear whether all hyperfinite (not finite) unions of elements in $\mathcal{U}$ are elements of $\overline{\mathcal{F}}$.

The following lemma provides a simple sufficient condition under which $\mathcal{H} \subset \overline{\mathcal{F}}$. Define $\mathcal{F}_{0}^{\prime}=\left\{F \in \mathcal{F}_{0}: F\right.$ intersects at least two elements in $\left.\mathcal{G}_{0}\right\}$.

Theorem 4.1. Suppose $\mathcal{G} \subset \overline{\mathcal{F}}$ and $\mu\left(\bigcup \mathcal{F}_{0}^{\prime}\right) \approx 0$. Then $\mathcal{H} \subset \overline{\mathcal{F}}$.

Proof. Let $\mathcal{F}_{1}=\mathcal{F}_{0} \backslash \mathcal{F}_{0}^{\prime}$. Pick $H \in \mathcal{H}$ and, without loss of generality, assume that $H=\bigcup_{i=1}^{K}\left(A_{i} \cap B_{i}\right)$ where $A_{i} \in \mathcal{F}, B_{i} \in \mathcal{G}$ and $K \in{ }^{*} \mathbb{N}$. Let $\mathcal{V}=\left\{A_{i} \cap B_{i}\right.$ : $i \leq K\}$. Then $H=H_{1} \cup H_{2}$ where $H_{1}=\bigcup\left\{\left(A_{i} \cap B_{i}\right) \in \mathcal{V}: A_{i} \in \mathcal{F}_{0}^{\prime}\right\}$ and $H_{2}=\bigcup\left\{\left(A_{i} \cap B_{i}\right) \in \mathcal{V}: A_{i} \in \mathcal{F}_{1}\right\}$. Clearly, $H_{1}$ is a subset of $\bigcup \mathcal{F}_{0}^{\prime}$. As $\mu\left(\bigcup \mathcal{F}_{0}^{\prime}\right) \approx 0$, by the completeness of Loeb measure, $H_{1}$ is Loeb measurable and $\bar{\mu}\left(H_{1}\right)=0$. Note that, for every element $F \in \mathcal{F}_{1}$, there exists an unique $G \in \mathcal{G}_{0}$ such that $F \cap G \neq \emptyset$ and hence $F \subset G$. Thus, for every $A_{i} \in \mathcal{F}_{1}, A_{i} \cap B_{i}$ is either $A_{i}$ or $\emptyset$. Thus, we know that $H_{2} \in \mathcal{F}$. Hence we conclude that $H \in \overline{\mathcal{F}}$, completing the proof.

In the remainder of this section, we show that $\mathcal{F}_{0}^{\prime}$ contains an internal subset $\mathscr{B}$ such that

(1) $\mu(\bigcup \mathscr{B}) \approx 0$;

(2) $\bigcup \mathscr{B} \cap G \neq \emptyset$ for every $G \in \mathcal{G}_{0}^{\prime}$.

Let us start with the following lemma.

Lemma 4.2. Let $(\Omega, \mathcal{F}, \mu)$ be a hyperfinite probability space and let $\mathcal{G}$ be a hyperfinite algebra on $\Omega$ such that $\mathcal{G} \subset \overline{\mathcal{F}}$. Let $\mathscr{B}$ be an internal subset of $\mathcal{F}_{0}^{\prime}$. Suppose there exists $G_{1} \in \mathcal{G}$ such that both $G_{1}$ and $\Omega \backslash G_{1}$ intersect every element in $\mathscr{B}$. Then $\mu(\bigcup \mathscr{B}) \approx 0$.

Proof. Let $F_{G_{1}}=\bigcap\left\{F \in \mathcal{F}: G_{1} \subset F\right\}$. Since $G_{1}$ intersects every element in $\mathscr{B}$, we know that $\bigcup \mathscr{B} \subset F_{G_{1}}$. Thus, by the Loeb measurability of $G_{1}$, we know that $\bigcup \mathscr{B} \backslash G_{1}$ has Loeb measure 0 . Similarly, we can conclude that $\bigcup \mathscr{B} \backslash\left(\Omega \backslash G_{1}\right)$ has Loeb measure 0 . Thus, we can conclude that $\mu(\bigcup \mathscr{B}) \approx 0$.

In what follows, let us write $\mathcal{G}_{0}^{\prime}=\left\{G \in \mathcal{G}_{0}:\left(\exists F \in \mathcal{F}_{0}^{\prime}\right)(G \cap F \neq \emptyset)\right\}$. 
Theorem 4.3. Let $(\Omega, \mathcal{F}, \mu)$ be a hyperfinite probability space and let $\mathcal{G}$ be an internal algebra on $\Omega$ such that $\mathcal{G} \subset \overline{\mathcal{F}}$. Suppose $\mathcal{F}_{0}^{\prime}$ is non-empty. Then there exists an internal $\mathscr{B} \subset \mathcal{F}_{0}^{\prime}$ such that

(A) $\mu(\bigcup \mathscr{B}) \approx 0$;

(B) $\bigcup \mathscr{B} \cap G \neq \emptyset$ for every $G \in \mathcal{G}_{0}^{\prime}$.

Proof. We now find a hyperfinite sequence $G_{1}, \ldots, G_{K}$ using the following algorithm, which takes hyperfinitely many steps to complete.

(1) Pick any element $G_{1} \in \mathcal{G}_{0}^{\prime}$ and let $\mathscr{B}_{G_{1}}=\left\{C \in \mathcal{F}_{0}^{\prime}: C \cap G_{1} \neq \emptyset\right\}$.

(2) For $k \geq 2$, assuming $G_{1}, \ldots, G_{k-1}$ have already been chosen, pick $G_{k} \in \mathscr{B}_{0}^{\prime}$ such that $G_{k} \cap \bigcup_{i=1}^{k-1} \bigcup \mathscr{B}_{G_{i}}=\emptyset$.

We continue this process until no $G_{k}$ as in Item (2) can be found, which must happen after a hyperfinite number of steps. Suppose that $K \in{ }^{*} \mathbb{N}$ is such that $G_{K}$ is the last element of $\mathcal{G}_{0}^{\prime}$ found by our algorithm, and consider the set $\bigcup_{i=1}^{K} \mathscr{B}_{G_{i}}$. Note that $\bigcup_{i=1}^{K} \mathscr{B}_{G_{i}}$ is an internal subset of $\mathcal{F}_{0}^{\prime}$, since by construction, the sequence $G_{1}, \ldots, G_{K}$ is internal.

Claim 4.4. $\mu\left(\bigcup \bigcup_{i=1}^{K} \mathscr{B}_{G_{i}}\right) \approx 0$.

Proof. It is clear that $\bigcup_{i=1}^{K} G_{i}$ intersects with every element in $\bigcup_{i=1}^{K} \mathscr{B}_{G_{i}}$. Pick $A \in \bigcup_{i=1}^{K} \mathscr{B}_{G_{i}}$. By construction, there exists an unique $1 \leq j \leq K$ such that $A \in \mathscr{B}_{G_{j}}$. As $A \in \mathcal{F}_{0}^{\prime}$, we conclude that $A \cap\left(\Omega \backslash G_{j}\right) \neq \emptyset$. For every $i \neq j$, we also have $A \cap\left(\Omega \backslash G_{i}\right) \neq \emptyset$, hence we conclude that both $\bigcup_{i=1}^{K} G_{i}$ and its complement intersect with every element of $\bigcup_{i=1}^{K} \mathscr{B}_{G_{i}}$. Thus, by Lemma 4.2, we have $\mu\left(\bigcup \bigcup_{i=1}^{K} \mathscr{B}_{G_{i}}\right) \approx 0$.

Claim 4.5. $\bigcup \bigcup_{i=1}^{K} \mathscr{B}_{G_{i}} \cap G \neq \emptyset$ for every $G \in \mathcal{G}_{0}^{\prime}$.

Proof. Suppose not. Then there exists some $G_{0}$ such that

$$
\bigcup_{i=1}^{K} \bigcup \mathscr{B}_{G_{i}} \cap G_{0}=\bigcup \bigcup_{i=1}^{K} \mathscr{B}_{G_{i}} \cap G_{0}=\emptyset .
$$

Thus, $G_{0}$ shall be added into the algorithm and the length of the algorithm would be $K+1$. This contradicts with the fact that our algorithm ends in $K$ steps.

Combining Claim 4.4 and Claim 4.5, we have the desired result.

We conclude the paper with the following open problem.

Open Problem 3. Let $\mathcal{M}=(\Omega, \mathcal{F}, \mu)$ and $\mathcal{N}=(\Omega, \mathcal{G}, \nu)$ be two hyperfinite probability spaces. If $\bar{\mu}\left(\bigcup \mathcal{F}_{0}^{\prime}\right)>0$, is it possible that $\mathcal{H} \subset \overline{\mathcal{F}}$ ?

\section{REFERENCES}

[AHKFL86] Sergio Albeverio, Raphael Høegh-Krohn, Jens Erik Fenstad, and Tom Lindstrøm, Nonstandard methods in stochastic analysis and mathematical physics, Pure and Applied Mathematics, vol. 122, Academic Press, Inc., Orlando, FL, 1986. MR859372

[AKS03] M. Ali Khan and Yeneng Sun, Exact arbitrage and portfolio analysis in large asset markets, Econom. Theory 22 (2003), no. 3, 495-528, DOI 10.1007/s001990200328. MR 2003831

[And76] Robert M. Anderson, A non-standard representation for Brownian motion and Itô integration, Israel J. Math. 25 (1976), no. 1-2, 15-46, DOI 10.1007/BF02756559. MR 464380 
[And78] Robert M. Anderson, An elementary core equivalence theorem, Econometrica 46 (1978), no. 6, 1483-1487, DOI 10.2307/1913840. MR513701

[And82] Robert M. Anderson, Star-finite representations of measure spaces, Trans. Amer. Math. Soc. 271 (1982), no. 2, 667-687, DOI 10.2307/1998904. MR654856

[And85] Robert M. Anderson, Strong core theorems with nonconvex preferences, Econometrica 53 (1985), no. 6, 1283-1294, DOI 10.2307/1913208. MR809911

[And88] Robert M. Anderson, The second welfare theorem with nonconvex preferences, Econometrica 56 (1988), no. 2, 361-382, DOI 10.2307/1911076. With an appendix by Anderson and Andreu Mas-Colell. MR935630

[And91] Robert M. Anderson, Nonstandard analysis with applications to economics, Handbook of mathematical economics, Vol. IV, Handbooks in Econom., vol. 1, NorthHolland, Amsterdam, 1991, pp. 2145-2208. MR.1207198

[ADS18] Robert M. Anderson, Haosui Duanmu, and Aaron Smith, Mixing times and hitting times for general Markov processes, 2018. Submitted.

[AR08] Robert M. Anderson and Roberto C. Raimondo, Equilibrium in continuous-time financial markets: endogenously dynamically complete markets, Econometrica $\mathbf{7 6}$ (2008), no. 4, 841-907, DOI 10.1111/j.1468-0262.2008.00861.x. MR2433482

[AR78] Robert M. Anderson and Salim Rashid, A nonstandard characterization of weak convergence, Proc. Amer. Math. Soc. 69 (1978), no. 2, 327-332, DOI 10.2307/2042621. MR 480925

[BR74] Donald J. Brown and Abraham Robinson, The cores of large standard exchange economies, J. Econom. Theory 9 (1974), no. 3, 245-254, DOI 10.1016/00220531(74)90050-7. MR475731

[BR75] Donald J. Brown and Abraham Robinson, Nonstandard exchange economies, Econometrica 43 (1975), 41-55, DOI 10.2307/1913412. MR443867

[CHLS19] Yi-Chun Chen, Wei He, Jiangtao Li, and Yeneng Sun, Equivalence of stochastic and deterministic mechanisms, Econometrica 87 (2019), no. 4, 1367-1390, DOI 10.3982/ECTA14698. MR3994274

[DRW18] Haosui Duanmu, J.S. Rosenthal, and William Weiss, Ergodicity of markov processes via non-standard analysis, 2018. Memoirs of the American Mathematical Society, to appear.

[DR18] Haosui Duanmu and Daniel M. Roy, On extended admissible procedures and their nonstandard bayes risk, 2018. The Annals of Statistics, to appear.

[DQS18] Darrell Duffie, Lei Qiao, and Yeneng Sun, Dynamic directed random matching, J. Econom. Theory 174 (2018), 124-183, DOI 10.1016/j.jet.2017.11.011. MR3759045

[DS07] Darrell Duffie and Yeneng Sun, Existence of independent random matching, Ann. Appl. Probab. 17 (2007), no. 1, 386-419, DOI 10.1214/105051606000000673. MR2292591

[Emm84] David W. Emmons, Existence of Lindahl equilibria in measure theoretic economies without ordered preferences, J. Econom. Theory 34 (1984), no. 2, 342-359, DOI 10.1016/0022-0531(84)90148-0. MR771008

[Kei84] H. Jerome Keisler, An infinitesimal approach to stochastic analysis, Mem. Amer. Math. Soc. 48 (1984), no. 297, x+184, DOI 10.1090/memo/0297. MR732752

[KS04] H. Jerome Keisler and Yeneng Sun, A metric on probabilities, and products of Loeb spaces, J. London Math. Soc. (2) 69 (2004), no. 1, 258-272, DOI 10.1112/S0024610703004794. MR2025340

[Kha74] M. Ali Khan, Some remarks on the core of a "large" economy, Econometrica 42 (1974), 633-642, DOI 10.2307/1913934. MR452542

[Kha76] M. Ali Khan, Oligopoly in markets with a continuum of traders: an asymptotic interpretation, J. Econom. Theory 12 (1976), no. 2, 273-297, DOI 10.1016/00220531(76)90078-8. MR411572

[KR75] M. Ali Khan and S. Rashid, Nonconvexity and Pareto optimality in large markets, Internat. Econom. Rev. 16 (1975), 222-245, DOI 10.2307/2525895. MR 408768

[AKRS97] M. Ali Khan, Kali P. Rath, and Yeneng Sun, On the existence of pure strategy equilibria in games with a continuum of players, J. Econom. Theory 76 (1997), no. 1, 13-46, DOI 10.1006/jeth.1997.2292. MR 1477341 
[KS99] M. Ali Khan and Yeneng Sun, Non-cooperative games on hyperfinite Loeb spaces, J. Math. Econom. 31 (1999), no. 4, 455-492, DOI 10.1016/S0304-4068(98)00031-7. MR.1688400

[Lin90] Tom Lindstrøm, Brownian motion on nested fractals, Mem. Amer. Math. Soc. 83 (1990), no. 420, iv+128, DOI 10.1090/memo/0420. MR988082

[Lin04] Tom Lindstrøm, Hyperfinite Lévy processes, Stoch. Stoch. Rep. 76 (2004), no. 6, 517-548, DOI 10.1080/10451120412331315797. MR2100020

[Loe75] Peter A. Loeb, Conversion from nonstandard to standard measure spaces and applications in probability theory, Trans. Amer. Math. Soc. 211 (1975), 113-122, DOI 10.2307/1997222. MR.390154

[Loe76] Peter A. Loeb, Applications of nonstandard analysis to ideal boundaries in potential theory, Israel J. Math. 25 (1976), no. 1-2, 154-187, DOI 10.1007/BF02756567. MR.457757

[Per81] Edwin Perkins, A global intrinsic characterization of Brownian local time, Ann. Probab. 9 (1981), no. 5, 800-817. MR628874

[Ras78] Salim Rashid, Existence of equilibrium in infinite economies with production, Econometrica 46 (1978), no. 5, 1155-1164, DOI 10.2307/1911440. MR508689

[Rau07] Michael T. Rauh, Nonstandard foundations of equilibrium search models, J. Econom. Theory 132 (2007), no. 1, 518-529, DOI 10.1016/j.jet.2004.07.011. MR2285619

[Sti97] Maxwell B. Stinchcombe, Countably additive subjective probabilities, Rev. Econom. Stud. 64 (1997), no. 1, 125-146, DOI 10.2307/2971743. MR 1433545

[Sto86] Andreas Stoll, A nonstandard construction of Lévy Brownian motion, Probab. Theory Relat. Fields 71 (1986), no. 3, 321-334, DOI 10.1007/BF01000208. MR824706

[Sun16] Xiang Sun, Independent random partial matching with general types, Adv. Math. 286 (2016), 683-702, DOI 10.1016/j.aim.2015.08.030. MR3415693

[Sun96] Yeneng Sun, Hyperfinite law of large numbers, Bull. Symbolic Logic 2 (1996), no. 2, 189-198, DOI 10.2307/421109. MR 1396854

[Sun99] Yeneng Sun, The complete removal of individual uncertainty: multiple optimal choices and random exchange economies, Econom. Theory 14 (1999), no. 3, 507544, DOI 10.1007/s001990050338. MR 1725660

Department of Economics, University of California, Berkeley, California 94720

Department of Economics, University of California, Berkeley, California 94720

Kurt Gödel Research Center, University of Vienna, Universitätssring 1, 1010 Vienna, Austria

Department of Mathematics, University of Toronto, Toronto, Ontario m5s 1A1, Canada 